\title{
Structure and Dynamics of an Eruptive Prominence on the Quiet Sun
}

\section{Yingna Su, Katharine K. Reeves, Patrick McCauley, Adriaan A. van Ballegooijen and Edward E. DeLuca}

Harvard-Smithsonian Center for Astrophysics, 60 Garden street, Cambridge, MA 02138, USA

email: ysu@cfa.harvard.edu

\begin{abstract}
We present preliminary results on the investigation of one polar crown prominence that erupted on 2012 March 11. This prominence is viewed at the east limb by SDO/AIA and displays a simple vertical-thread structure. A bright U-shape (double horn-like) structure is observed surrounding the upper portion of the prominence before the eruption and becomes more prominent during the eruption. When viewed on the disk, STEREO_B shows that this prominence is composed of series of vertical threads and displays a loop-like structure during the eruption. We focus on the magnetic support of the prominence by studying the structure and dynamics before and during the eruption using observations from SDO and STEREO. We will also present preliminary DEM analysis of the cavity surrounding the prominence.
\end{abstract}

Keywords. Sun: coronal mass ejections (CMEs), Sun: filament eruptions, Sun: magnetic fields.

\section{Observations}

What is the magnetic structure supporting hedgerow prominences? Are they supported by the dips in a twisted flux rope or tangled fields in a current sheet (e.g., van Ballegooijen \& Cranmer 2010, Berger 2012, Fan 2012, and references therein)? To address this question, we study a polar crown prominence that erupted on 2012 March 11.

Figure 1 shows AIA emission maps of the filament and surroundings at different temperature bands before the eruption. The maps are derived from the DEMs calculated using six AIA EUV channels in each pixel. The filament contains vertical threads surrounded with a bright inverted triangle structure and a bright U-shape structure on top (Figures 1a-1b). The DEM study suggests that the bright emission surrounding the vertical threads has relatively low temperature $(<1 \mathrm{MK})$, and the cavity has lower density than the background corona. There is also a hot core (concentrated substructure at 2-3 MK in Figure 1c) located on top of the threads within the cavity.

Figure 2 shows AIA and STEREO_B observations of the erupting prominence on 2012 March 12. The filament displays a slow-rise phase $\left(\mathrm{V} \sim 0.2 \mathrm{~km} \mathrm{~s}^{-1}\right)$ and a fast-rise phase $\left(\mathrm{V} \sim 27 \mathrm{~km} \mathrm{~s}^{-1}\right)$ starting around 17:00 UT and 22:40 UT on March 11, 2012, respectively (Figure 2b). Upon exiting the AIA field of view, the filament has velocity of 64.8 $\mathrm{km} \mathrm{s}^{-1}$, and acceleration is $0.02 \mathrm{~km} \mathrm{~s}^{-2}$. The eruption is associated with a partial halo CME with a linear speed of $638 \mathrm{~km} \mathrm{~s}^{-1}$ as provided by the SOHO LASCO CME catalog. The timing of the filament appearance in LASCO suggests additional acceleration. AIA $171 \AA$ observations show that during the eruption, more and more bright U-shape structures appear to go across the vertical filament threads starting from the upper to the lower portion. The filament displays an asymmetric arch-like structure during the eruption as shown in STEREO_B (Figure 2f). We also observe brightenings of prominence material at the inner edge of the filament arch. A clear U-shape structure is also seen at $171 \AA$ in STEREO_B during eruption (Figure $2 \mathrm{~g}$ ). More comprehensive analysis 

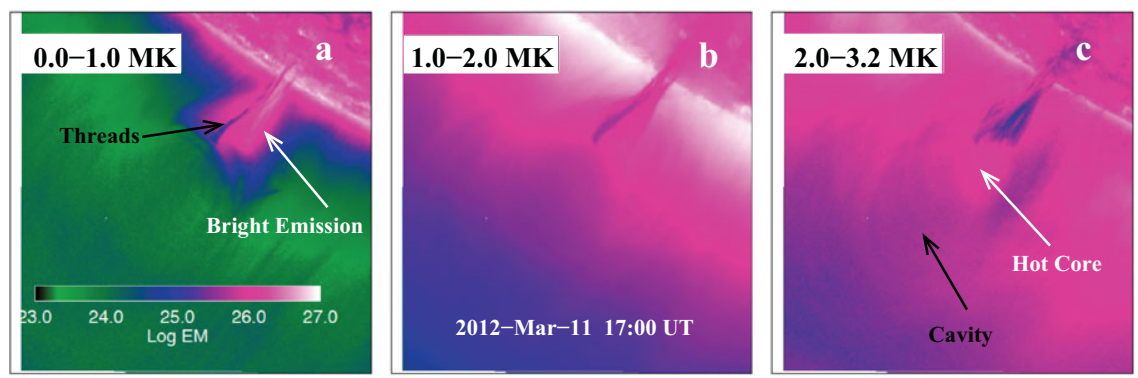

Figure 1. AIA Emission Measure maps at 17:00 UT on 2012 March 11.
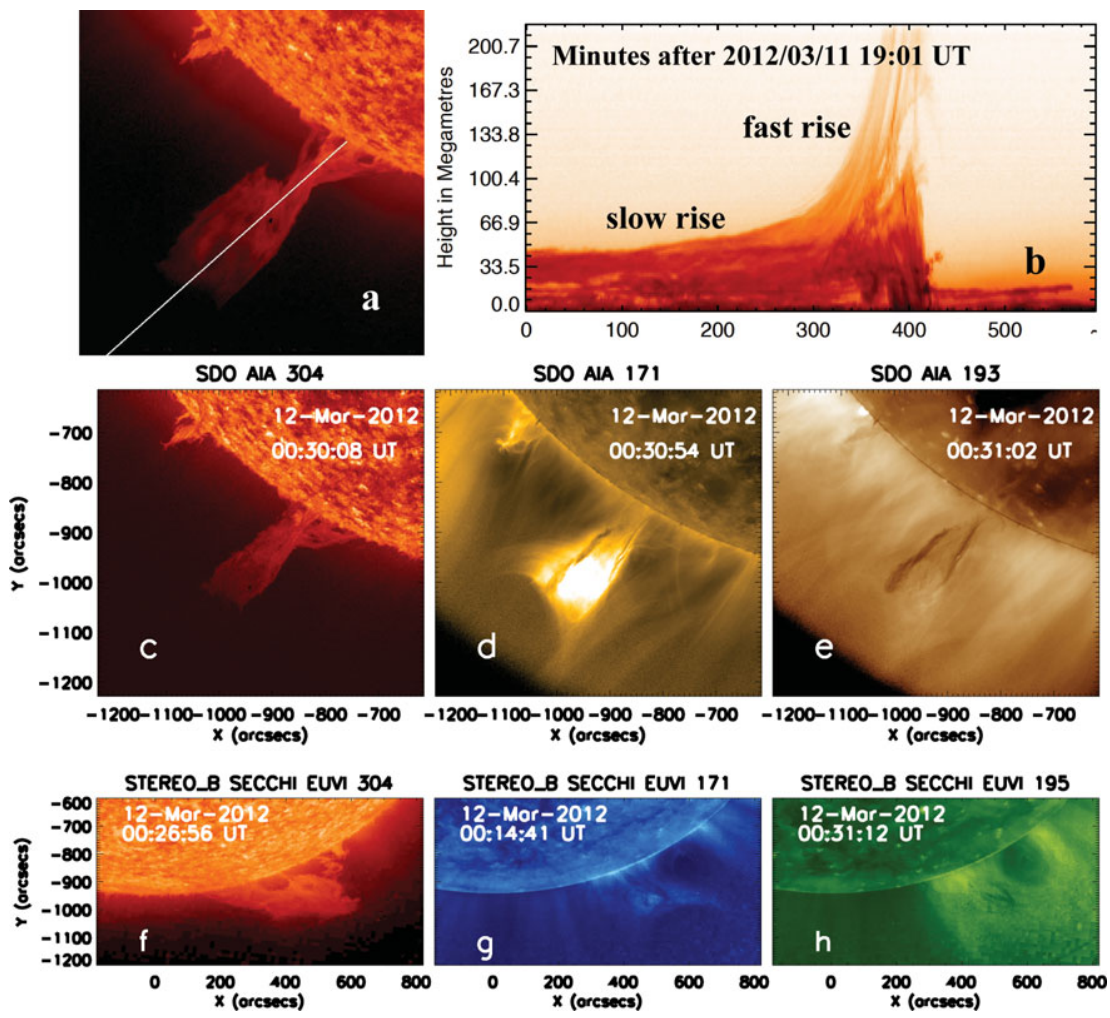

Figure 2. SDO and STEREO_B observations of the erupting prominence on March 12, 2012.

of the magnetic structure and thermodynamics of the erupting prominence is under investigation. For detailed column density study of this prominence, please see the paper by McCauley et al. in this proceedings.

Acknowledgements: This project is supported by NASA grant NNX12AI30G as well as NASA contract SP02H1701R from LMSAL to SAO.

\section{References}

Berger, T. 2012, Second ATST-EAST Meeting: Magnetic Fields from the Photosphere to the Corona., 463, 147

Fan, Y. 2012, ApJ, 758, 60

van Ballegooijen, A. A. \& Cranmer, S. R. 2010, ApJ, 711, 164 